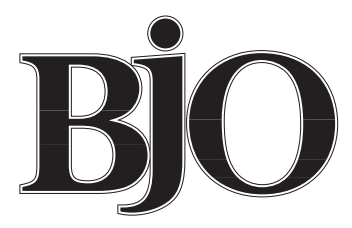

British Journal of Ophthalmology

The eye is the window to the brain, the soul, and now perhaps even the future, with the exciting publication by Chowers et al in this month's issue of the BfO (p 991). The authors give us a view of the future as they elegantly demonstrate that gene transfer into blood vessels is possible in a rat model of retinopathy of prematurity (ROP). The future may be close at hand, because the eye once again offers an ideal organ to study the effects of this new therapy. ROP, a developmental disease of retinal angiogenesis in premature infants, is also an optimal and important disease to study potential therapeutic effects of gene therapy.

To date, the view of future gene therapy has been through hazy media at best. Despite incredible advances in developmental biology and molecular genetics during the past several decades, efforts to utilise these advances, and to deliver therapeutic genes to target organs or structures, have often been disappointing. The reason for disappointment usually hinges on delivery and expression of the therapeutic gene to the desired target. A vector must be used, one that transports a gene or genes to the target. The vector should be innocuous, sustainable, and, of course, able to deliver a genetic sequence that can be inserted into target cells' DNA. Thus the vector also must be able to cross cell membranes and survive an environment that eschews anything foreign. While outside the cell, the vector must be able to survive the host's immune system. It is this latter issue - the host immune system - that arguably poses the most formidable challenge to gene therapy. Viral vectors used for gene therapy are quickly recognised by the host immune system and eradicated.

With current technology, the ideal disease to study gene therapy should be developmental (occurring only during a short time period) and should occur in an immunologically privileged part of the body. ROP is one such disease. It occurs during a narrow window of time-the length of time from the onset of pathological neovascularisation to devastating retinal detachment is usually measured in weeks. Even if a viral vector only survives a few days or weeks, it still could effectively provide genetic material long enough to affect the disease. And ROP occurs inside the eye where the immune system is relatively inactive. Clearly, the authors have chosen a disease where gene therapy has a fighting chance to succeed.

ROP is also a disease in need of better therapy. It occurs in premature infants and remains a leading cause of vision impairment in children. ${ }^{1}$ Advances in neonatology allowing survival of the smallest infants contribute to the incidence of ROP. Although cryosurgery and laser retinal ablation of avascular retina improve chances of a favourable outcome, approximately $30 \%$ of infants with threshold ROP experience a retinal detachment and blindness. ${ }^{2}$ More than $80 \%$ of infants who develop threshold ROP develop visual acuity of $20 / 40$ or worse, and even when the retina remains attached. ${ }^{3}$ Other blinding eye diseases are also caused by pathological neovascularisation, including diabetic retinopathy and age related macular degeneration.

Gene therapy mediated regulation of cytokines and growth factors known to be involved in ROP would be a promising approach if proved to be feasible in animal models. Cytokines such as vascular endothelial growth factor (VEGF), basic fibroblast growth factor (bFGF) and insulin-like growth factor (IGF-1), hypoxia inducing factor (HIF-1), and angiopoietin 2 may have roles in ROP. Therefore, the targeting and regulation of such factors by gene therapy is a realistic goal for developing newer and better ROP treatments. In their studies, Chowers et al tested retrovirus, adenovirus and herpes virus based vectors, shuttling a $\beta$ galactosidase reporter gene for expression in retinal blood vessels in rodents undergoing oxygen induced retinal neovascularisation. Interestingly, they found that adenovirus offered the best efficiency in expression in retinal blood vessels compared with all the other vectors. Moreover, the adenovirus expression was specific to the blood vessels of the inner retina and did not appear to be expressed in the deeper neural retina.

These studies indicate that adenoviral vectors can be expressed in retinal blood vessels and therefore may provide a feasible means for shuttling genes of interest into retinal blood vessels undergoing neovascularisation. They also suggest that adenovirus can be targeted to specific areas of the eye, and might then minimise non-specific effects through collateral tissues.

Non-specific effects could be very problematic even when collateral tissues are not directly affected by gene therapy. Somehow, gene therapy for ROP will have to limit pathological retinal neovascularisation while permitting the normal process of retinal angiogenesis. The sequence of molecular events leading to development of blood vessels is complicated, with some effects of cytokines occurring at more remote ocular sites. In fact, ROP is more 
than simply a disease of retinal angiogenesis. Myopia is more common, ${ }^{4}$ visual acuity is usually diminished, and cicatricial changes brought about by the ROP disease process may lead to glaucoma.

Here the view of future gene therapy for ROP becomes obfuscated. Future researchers must carefully avoid these collateral effects and should consider the developmental nature of the disease. It would be a tragedy to halt pathological neovascularisation only to find additional, unwanted, downstream effects such as altered photoreceptor cell development or downregulation of factors that contribute to healthy blood vessel formation, such as pericyte growth and survival. The future requires more basic science work. Until we know more detail about the molecular events that cause ROP, and their timing, it will be unsafe to intervene in human ROP. In neovascular diseases occurring in mature systems-for example, diabetic retinopathy, the future could be closer.

The advance reported in the $B f O$ this month moves us closer to a better treatment for neovascular eye disease. It is now possible to see shape and form in the future, but details remain obscure. We could well have a method to deliver genes to retinal vessels, but we need to learn which genes to deliver and when to deliver them, before embarking on treatment of human disease. This much is very clear: gene therapy has a future in blinding eye disease. Adenovirus vectors appear to effectively transfer genes into blood vessels in the eye.

Dr Good is supported by a grant from the National Institutes of Health EY00384

Dr Gendron is supported by a grant from the National Institutes of Health EY12827.

WILLIAM V GOOD

Smith-Kettlewell Eye Research Institute, San Francisco, California, USA

ROBERT L GENDRON

Cincinnati Childrens Hospital, Cincinnati, Ohio, USA

Correspondence to: William V Good, MD, Smith-Kettlewell Eye Research Institute, 2318 Filmore Strett, San Francisco, CA 94115, USA

Good@ski.org

1 Steinkuller PG, Du L, Gilbert C, et al. Childhood blindness. I Aapos 1999;3:26-32.

2 Multicenter Trial of Cryotherapy for Retinopathy of Prematurity. Preliminary results. Cryotherapy for Retinopathy of Prematurity Cooperative Group. Arch Ophthalmol 1988;106:471-9.

3 Multicenter Trial of Cryotherapy for Retinopathy of Prematurity. Snellen visual acuity and structural outcome at $5 \frac{1}{2}$ years after randomization. Cryotherapy for Retinopathy of Prematurity Cooperative Group. Arch Ophthalmol 1996;114:417-24.

4 Quinn GE, Dobson V, Kivlin J, et al. Prevalence of myopia between 3 months and $5 \frac{1}{2}$ years in preterm infants with and without retinopathy of prematurity. Cryotherapy for Retinopathy of Prematurity Cooperative Group. Ophthalmology 1998;105:1292-300.

\section{Optic disc changes following trabeculectomy}

The reversal of optic disc cupping following pressure reduction by medical or surgical means has long been recognised, particularly in congenital and juvenile glaucoma. Less clear cut is whether this reversal is accompanied by any improvement in visual function. The matter is, obviously, an important one and there are some reports suggesting that an improvement may occur. If so this presumably is due to recovery of neuronal function which has only temporarily been interrupted. Although it is of great importance to know how extensive this process may be, only studies conducted with great care and rigour are likely to tell this conclusively.

The precise pathological mechanism underlying any reversal is also important. Presumably there is reversal of bowing of the lamina but definitive proof of this is difficult. If there is in fact any recovery of function this may be due to a neuronal change although it is doubtful how this would occur, except by reversal of changes which are still transitory.

In a paper in the current issue of the BfO (p 956), Kotecha and coworkers have attempted to solve some of these contentious problems. They have identified "significant" increases in rim volume at 2 years following surgical operations which produced a fall in pressure of around $30 \%$. Some change in maximum cup depth was demonstrated. This was "significant" at 1 year but not at 2 years. The changes seem small. A change in rim volume suggests something more than just a lessening of bowing of the lamina. Although all patients had 24-2 threshold Humphrey visual field tests, no results of these are given so there is no comment regarding visual function. Perhaps a later paper will tell us more.

The results given hardly seem likely to be the basis for much recovery of lost visual function in glaucoma but they do suggest that a reduction in intraocular pressure to the low normal range will halt the glaucomatous process evident at the optic nerve head. However, it may still be too early to paraphrase the slogan of a recent US presidential election "It's the economy stupid" to "It's the pressure stupid."

ANNE M V BROOKS

BILL GILLIES

Royal Victorian Eye and Ear Hospital, East Melbourne,

Victoria 3002, Australia

amvbrooks@bigpond.com.au 


\section{Getting our journals to developing countries}

For some years now it has been our policy to give free subscriptions to our journals to applicants from countries in the developing world. However, in practice this has had its difficulties. Many developing countries have either poor or non-existent postal services and granting a print subscription can often be problematic and expensive-the marginal cost of sending the British fournal of Ophthalmology to Africa is around $£ 25$ each year.

An editorial in $B M F$ sets out the arguments very clearly. ${ }^{1}$ We know that the gap between the rich and poor countries is widening. While those of us in the developed world have information overload, the developing countries have bare library shelves. The internet gives us the opportunity to narrow the gap.

The marginal cost of giving access to the electronic edition of the $B F O$ is close to zero.

What is more, those in resource-poor countries can access electronic journals at exactly the same time as those in the developed world. Even better, they can access what is relevant rather than what was provided, much of which wasn't relevant. Best of all, they can participate in the debate using the rapid response facility on the website in a way that was almost impossible with the slowness of print distribution.

Access to the electronic edition of the $B F O$ of will be provided free automatically to those from countries defined as poor under the human development index by the United Nations and the World Bank (www. worldbank.org/data/databytopic/class.htm). The BMA and several of our co-owning societies have made funds available for the installation of Digital Island on all our journal websites. This clever piece of software recognises where the user is coming from and will give unrestricted access to the whole website to users from those developing countries we choose to designate.

BMJ.com will continue to be free to those in the developing world whatever happens in the developed world.

The income that we get from resource-poor countries is minimal; and facilitating information supply should encourage development, improvement in health care, and eventually create a market.
The problem with this vision is the lack of access to the world wide web in the developing world. While tens of millions of people have access in the United States, it is only thousands in most African countries; and access in Africa is often painfully slow, intermittent, and hugely expensive relative to access in the United States (where it's often free). Power cuts happen every day in many resource-poor countries. Yet there's every reason to expect that access should increase dramatically. India currently has a million people with internet access, but this is expected to rise to 40 million within 5 years. Similarly, dramatic increases are expected in Nigeria. Technological developments like access to radio and the proliferation of satellites will render irrelevant the many problems of telephone access in Africa. Rapid progress will also be made because many international organisations such as Unesco, the British government, the World Bank, and the Bill and Melissa Gates Foundation are increasingly interested in helping improve information access in resource-poor countries.

The challenge will be sustainability. It is easy for donors to invest money and reap the rewards of short term success. But enhancing information flow will have no impact on health if projects continue only as long as their funding lasts. Information cannot be separated from the capacity of a healthcare system to work effectively over time. How is it possible to influence the context within which information will flow, the apparently intractable political, economic, and organisational constraints that disable rather than enable information to work for people? Publishers in the rich world have a part to play and we hope that by making access to the $B \mathcal{F O}$ online free to those in the developing world we are making our own small contribution.

ALEX WILLIAMSON

BMJ Publishing Group

1 Godlee F, Horton R, Smith R. Global information flow. BMf 2000;321:776-7. 